\title{
Contents of Volume 22
}

Gröbner-Shirshov Bases for Exceptional Lie Superalgebras



On Ordered rpp Semigroups with Max-idempotents

X.J. Guo, C.C. Ren, K.P. Shum $\ldots \ldots \ldots \ldots \ldots \ldots \ldots \ldots \ldots \ldots \ldots \ldots \ldots \ldots \ldots$

Normally Torsion-free Lexsegment Ideals

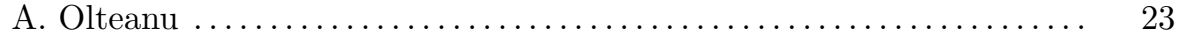

Gröbner-Shirshov Bases of Some Semigroup Constructions



Zariski Topologies for Coprime and Second Submodules

J. Abuhlail

Artinian Local Rings Whose Annihilating-ideal Graphs Are Star Graphs

H.Y. Yu, T.S. Wu, W.P. Gu

On the Structure of Graded Leibniz Algebras

A.J.C. Martín, J.M.S. Delgado

Minimal Free Resolutions of Zero-dimensional Schemes in $\mathbb{P}^{1} \times \mathbb{P}^{1}$

P. Bonacini, L. Marino .......................................

Zero Product Determined Jordan Algebras, II

M. Grašič

When Finitely Generated $\delta$-Supplemented Modules Are Supplemented

R. Tribak

Tameness and Artinianness of Graded Generalized Local Cohomology

Modules

M. Jahangiri, N. Shirmohammadi, Sh. Tahamtan

On the Auslander-Yoneda Algebras of Modules over $K[x] /\left(x^{n}\right)$

R.D. Zheng ...

Generalized Derivations with Periodic Values

K.-S. Liu

Some *-Clean Group Rings

Y.Y. Gao, J.L. Chen, Y.L. Li

Groups Whose Proper Subgroups of Infinite Rank Have Polycyclic

Conjugacy Classes

F. de Giovanni, M. Trombetti 
Harmonic Analysis of Radon Filtrations for $S_{n}$ and Its $q$-Analogue

$$
G L_{n}(q)
$$

M.F. Yáñez

On the Cover-Avoid Property of Injectors for Hartley Classes

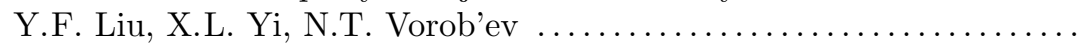

Homological Dimensions with Respect to a Semidualizing Module and

Tensor Products of Algebras

M. Salimi, E. Tavasoli, S. Yassemi

Sequentially Cohen-Macaulay Mixed Product Ideals

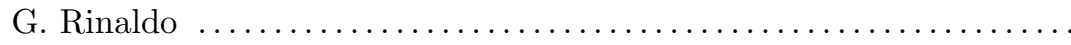

Representations of Code Vertex Operator Superalgebras

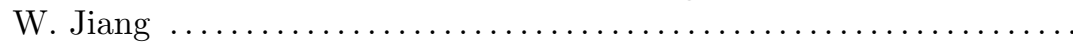

The $\sigma$-Derivations of $\mathbb{C}\left[x^{ \pm 1}, y^{ \pm 1}\right]$

G.A. Song, Y.Z. Wu, B. Xin

Gorenstein Flat Complexes with Respect to a Semidualizing Module

L. Liang, C.H. Yang . . . . . . . . . . . . . . . . . . . . . . .

On Skew Triangular Matrix Rings

M. Habibi, A. Moussavi, A. Alhevaz ......................

Universal Enveloping Algebras of Simple Symplectic Anti-Jordan Triple

Systems

M. Tvalavadze

On Huppert's Conjecture for Alternating Groups of Low Degrees

H.N. Nguyen, H.P. Tong-Viet, T.P. Wakefield ..................

Restricted Envelopes of Lie Superalgebras

L.P. Sun, W.D. Liu, X.C. Gao, B.Y. Wu

Skew-symmetric Elements in Nonlinear Involutions in Group Rings

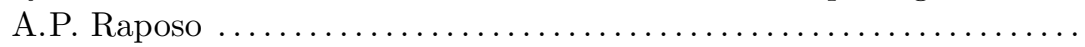

Growth in Infinite Groups of Infinite Subsets

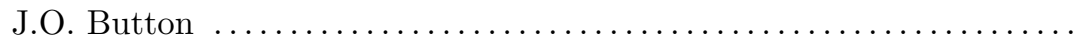

On $n$-Coherent Rings and $(n, d)$-Injective Modules

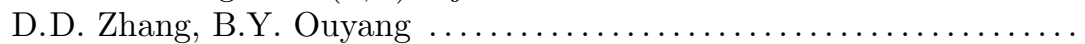

A Note on the Commutativity of Prime Near-rings

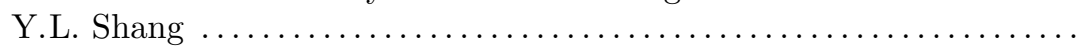

The Lie Conformal Algebra of a Block Type Lie Algebra



Pentavalent Symmetric Graphs of Order Twice a Prime Square



Standard Monomial Theory of RR Varieties




$\mathcal{W}(a, b)$ Lie Conformal Algebra and Its Conformal Module of Rank One

Y. Xu, X.Q. Yue

Strong Separativity over Regular Rings

H.Y. Chen

On Mixed Multiplicities of Good Filtrations

D.Q. Viet, L.V. Dinh

Finite Groups of Spencer Height $\leq 3$

W.B. Guo, D.P. Andreeva, A.N. Skiba

A Hua-like Theorem in Semirings

J. Pei, Y.Q. Guo, K.P. Shum

Finite Groups with Four Relative Commutativity Degrees

A. Erfanian, M. Farrokhi D.G.

Recognizing the Semiprimitivity of $\mathbb{N}$-graded Algebras via Gröbner Bases

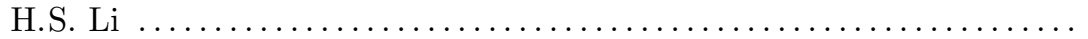

Gorenstein Injective and Injective Complete Cohomological Dimensions of Groups

A. Bahlekeh

Skew Derivations with Power Central Values on Commutators

H.-Y. Chen

Gröbner-Shirshov Basis of Quantum Groups

G. Yunus, Z.Z. Gao, A. Obul

Derivations and Automorphism Group of Original Deformative

Schrödinger-Virasoro Algebra

Q.F. Jiang, S. Wang

Periodic Algebras Generated by Groups

S. Albeverio, B.A. Omirov, U.A. Rozikov

On Non-commuting Sets in Certain Finite $p$-Groups

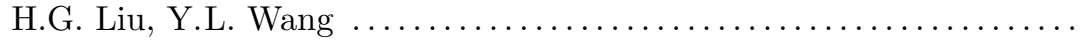

Braids with Trivial Simple Centralizer

U. Ali, F. Azam, I. Javaid, A. Haider

Indispensable Hibi Relations and Gröbner Bases

A.A. Qureshi

Quantizations of the Extended Affine Lie Algebra $\widetilde{\mathfrak{s l}_{2}\left(\mathbb{C}_{q}\right)}$

Y. Xu, J.B. Li

A Proof of Wedderburn's Theorem

J.A. Cuenca Mira

On Annihilator Ideals of Pseudo-differential Operator Rings

R. Manaviyat, A. Moussavi .............................. 
A Length Function for Weyl Groups of Extended Affine Root Systems of Type $A_{1}$

S. Azam, M. Nikouei

On Simple Connectedness of Minimal Representation-Infinite Algebras

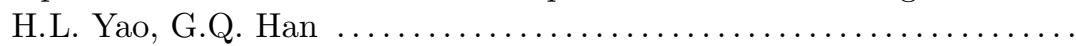

C3-Modules

I. Amin, Y. Ibrahim, M. Yousif

655

Ideals Whose First Two Betti Numbers Are Close

K. Borna, S.H. Hassanzadeh

671

Differential Graded Structures on Path Categories

F. Li, D.Z. Tan

Semi-topological Galois Theory

H.-Y. Liao, J.-H. Teh

Spanning Simplicial Complexes of Uni-Cyclic Graphs

I. Anwar, Z. Raza, A. Kashif

Non-degenerate Invariant Bilinear Forms on Leibniz Algebras

S. Wang, L.S. Zhu

Contents of Volume 22

Author Index for Volume 22 